\title{
ERRATUM
}

The Journal of Microbiology (2012) Vol. 50, No. 3, pp. 478-488 Copyright (C) 2012, The Microbiological Society of Korea

\section{Evaluation of the Efficacy of a Pre-pandemic H5N1 Vaccine (MG1109) in Mouse and Ferret Models}

\author{
Min-Suk Song ${ }^{1}$, Ho-Jin Moon ${ }^{2}$, Hyeok-il Kwon ${ }^{1}$, Philippe Noriel Q. Pascua ${ }^{1}$, Jun Han Lee ${ }^{1}$, \\ Yun Hee Baek ${ }^{1}$, Kyu-Jin $\mathrm{Woo}^{3}$, Juhee Choi ${ }^{3}$, Sangho Lee ${ }^{3}$, Hyunseung $\mathrm{Yoo}^{3}$, In gyeong $\mathrm{Oh}^{3}$, \\ Yeup Yoon ${ }^{3}$, Jong-Bok Rho ${ }^{4}$, Moon-Hee Sung ${ }^{4}$, Seung-Pyo Hong ${ }^{4}$, Chul-Joong Kim ${ }^{2}$, \\ and Young Ki Choi ${ }^{1 *}$ \\ ${ }^{1}$ College of Medicine and Medical Research Institute, Chungbuk National University, Cheongju 361-763, Republic of Korea \\ ${ }^{2}$ College of Veterinary Medicine, Chungnam National University, Daejeon 305-764, Republic of Korea \\ ${ }^{3}$ Mogam Biotechology Research Institute, Yongin 446-799, Republic of Korea \\ ${ }^{4}$ Bioleaders Corporation, Daejeon 305-764, Republic of Korea
}

In the article by Song et al. that appears in the Journal of Microbiology 2012; 50, 478-488. Page 478, the name of 7th author, Kyu-Jin Woo, should read as Gyu-Jin Woo 